\section{«Quand le passé résiste} à l'oubli»

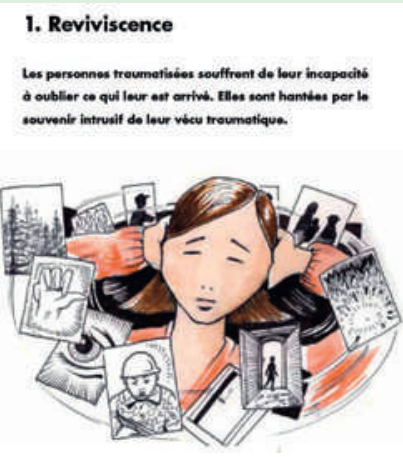

«Quand le passé résiste à l'oubli»: tel est le titre d'une brochure d'information de parution récente consacrée à l'état de stress post-traumatique, à ses séquelles et aux moyens de le surmonter. Il s'agit de la première publication que la Croix-Rouge suisse (CRS) consacre aux traumatismes consécutifs à la guerre, à la fuite, à la torture ou aux catastrophes naturelles. Grâce aux nombreux conseils formulés et à une liste étendue des interlocuteurs et ressources disponibles, cette brochure peut être d'une grande utilité aux victimes et à leur entourage.

(CRS)

\section{Welt-Lepra-Tag}

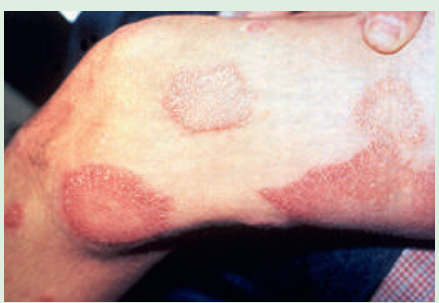

Am 27. Januar war der 60. WeltLepra-Tag. Immer noch erfahren jede Stunde 30 Menschen, dass sie Lepra haben, und alle 20 Minuten wird ein Kind diagnostiziert. Wenn Lepra nicht rechtzeitig behandelt wird, hat sie schwere körperliche Behinderungen und oft auch soziale Ausgrenzung zur Folge. Der WeltLepra-Tag machte auf die Not dieser Menschen aufmerksam. Die LepraMission Schweiz setzt sich dafür ein, dass alle von Lepra betroffenen Menschen ein Leben in Würde führen können, ein Leben mit denselben Rechten und Freiheiten wie alle anderen.

(Lepra-Mission Schweiz)

\section{Via sicura kommt in die Gänge}

Via sicura startet Anfang 2013 mit ersten Massnahmen, welche die Sicherheit auf Schweizer Strassen erhöhen sollen. Darunter sind solche gegen Raser vorgesehen, etwa die Einziehung von Motorfahrzeugen, sowie das Verbot von Radarwarnungen. Die zweite Tranche, die 2014 in Kraft treten soll, umfasst Massnahmen, die auf Verordnungsstufe konkretisiert werden müssen. Die wohl bedeutendste ist das faktische Alkoholverbot für Neulenkende jeden Alters. Auch Fahrerinnen und Fahrer von Lastwagen und Bussen dürfen fortan nur noch nüchtern hinter das Steuer. Fahren unter Alkoholeinfluss ist eine der häufigsten Unfallursachen. 18- bis 24-Jährige haben das höchste Risiko, hinter dem Steuer zu sterben. Sucht Schweiz begrüsst deshalb diese Massnahme, propagierte aber schon immer die Regel: Wer fährt, trinkt nicht.

(Sucht Schweiz)

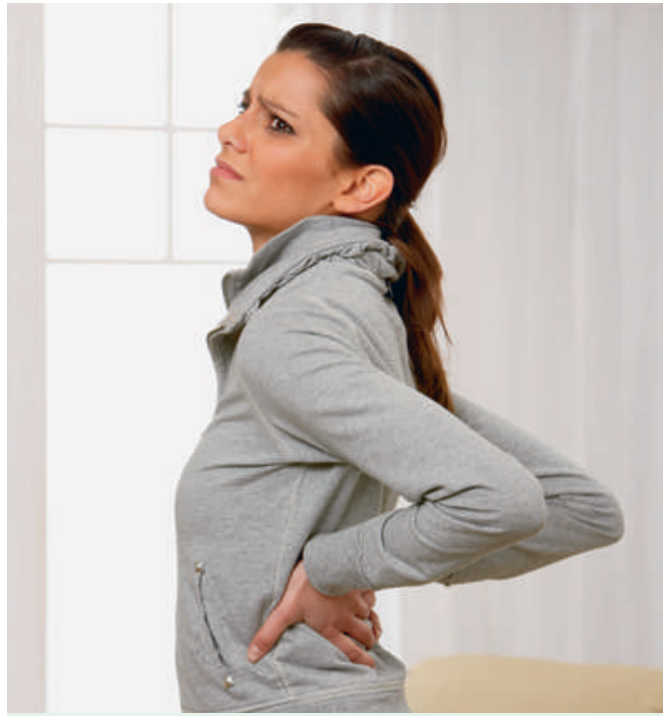

Ein Grund für Rückenschmerzen kann Morbus Bechterew sein.

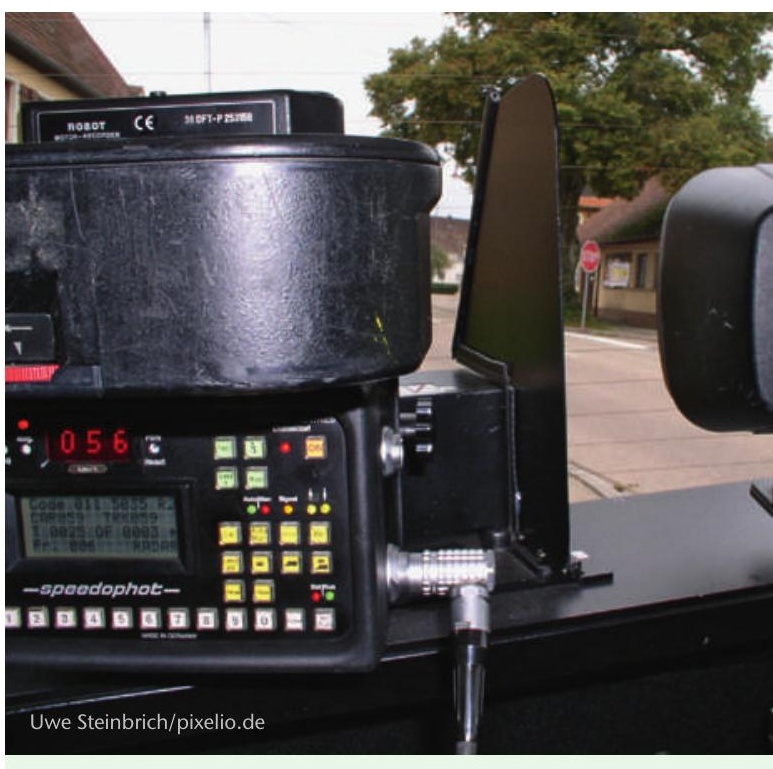

Warnungen vor Radarfallen sind künftig verboten.

\title{
«Höllische Rückenschmerzen»
}

Wenn «höllische Rückenschmerzen» den Schlaf rauben, kann dies auf eine entzündliche rheumatische Erkrankung hindeuten: Morbus Bechterew. Dies war die zentrale Botschaft der Online-Aufklärungskampagne der Schweizerischen Vereinigung Morbus Bechterew. Sie fokussierte sich auf die Zielgruppe der 18- bis 35-jährigen Männer und Frauen. Auf sie richtete sich auch Stil und Look der Medienstrategie. Die Anzeigen erschienen in 20 Minuten. Und die Botschaft kam an: Über 20000 Personen besuchten im Zeitraum der Kampagne die Plattform www.bechterew.ch. Und annähernd 10000 Personen klickten sich durch den Online-Diagnose-Test mit dem Ziel, die Wahrscheinlichkeit einer Erkrankung an Morbus Bechterew abzuklären. Je früher eine Diagnose erfolgt, desto wirkungsvoller lässt sich Morbus Bechterew behandeln.

(Rheumaliga Schweiz)

\section{Journée internationale du cancer de l'enfant}

La journée du 15 février est traditionnellement dédiée aux enfants touchés par le cancer. La Ligue suisse contre le cancer a lancé une action en vue de récolter des fonds en faveur de la recherche

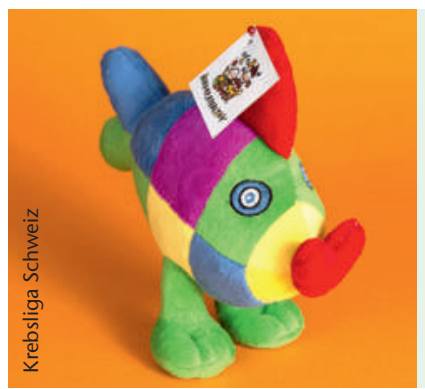

Le Scapafish est vendu dans les offices postaux pour soutenir la recherche des thérapies mieux adaptées aux jeunes. pédiatrique. Aujourd'hui, quatre enfants sur cinq peuvent être soignés avec succès. Mais ils souffrent souvent des séquelles de traitements trop agressifs pour leur organisme en pleine croissance. Afin de leur venir en aide, la Ligue suisse contre le cancer soutient de nombreux projets de recherche qui visent à mettre au point des thérapies plus ciblées et mieux adaptées aux jeunes patients. Jusqu'au 2 mars, le Scapafish sera encore vendu dans les offices postaux au prix de 19 francs 90 , dont 10 francs seront reversés à la Ligue. La somme servira à financer des projets pour améliorer la situation des enfants touchés.

(Ligue suisse contre le cancer) 